\title{
Simple TWRC technique by using optical fiber
}

\begin{abstract}
The metalized optical fiber tip was used to generate thermal wave instead of metal foil attached to a tube as in the case of conventional thermal wave resonance cavity (TWRC) technique. The liquid thermal diffusivity was obtained through scanning the cavity length by moving this tip with respect to pyroelectric detector in liquid media, in a thermally thick region. Three-dimensional treatment of the metalized fiber tip was reduced to onedimensional treatment using diameter of thermal wave generator relatively bigger than that of laser spot size. This applicability can be seen in two diameter size of optical fiber where the thermal diffusivity value of water obtained by this technique agrees with the values obtained by the conventional TWRC technique. This technique has a potential to be used in measuring thermal diffusivity of small liquid volume.
\end{abstract}

Keyword: Pyroelectric, Thermal wave, Thermal diffusivity, Optical fiber 